\title{
Overcoming SNR walls through macroscale features
}

\author{
Rahul Tandra \\ Anant Sahai \\ tandra@eecs.berkeley.edu sahai@eecs.berkeley.edu \\ Department of Electrical Engineering and Computer Sciences \\ University of California, Berkeley
}

\begin{abstract}
We consider the problem of robustly detecting the presence/absence of signals in low signal to noise ratio $(S N R)$ environments. Our previous results have shown the existence of thresholds called $S N R$ walls, below which robust detection becomes impossible due to uncertainties in the environment. These thresholds were shown to exist for many signals used in practice. In this paper we introduce the idea of macroscale features and show that they can be used to construct signals that evade $S N R$ walls. In particular, we examine a Gaussian mixture example and a noise-calibration based detection algorithm to show that this signal can be robustly detected in the presence of arbitrarily varying noise and arbitrarily varying finite-tap fading processes. Finally, we argue that there is tension between the primary user's capacity and the sensing delay experienced by the secondary users. We call this the capacity-delay tradeoff. We derive the capacity-delay tradeoff for the Gaussian example.
\end{abstract}

\section{INTRODUCTION}

Detection theory is a means to quantify the ability to distinguish signals from noise. It is well established and has applications in many fields like statistics, communications, and controls. Several signal detection algorithms have been proposed and are used widely in practice [1], [2], [3], [4]. Robustness to modeling uncertainties is a necessary attribute for any practical detection algorithm. That is, the performance of any detector must not deviate significantly from the idealized (with no uncertainties) performance in the presence of uncertainties. Robustness to uncertainties is easier to achieve at high signal to noise ratios (SNRs) as compared to low SNRs. The reason for this is that at high SNRs the distributions under both the hypotheses are sufficiently separated. So, even though the uncertainties in the environment perturb the individual distributions, they cannot completely mix the distributions. Hence, robustness is not very hard to achieve.

In this paper we consider the problem of robustly detecting the presence/absence of signals in low $S N R$ environments. The need to detect very weak signals arises in the context of cognitive radios trying to opportunistically reuse primary spectrum [5], [6], [7], [8]. The fundamental requirement of cognitive radios is not to cause harmful interference to the primary users of the spectrum. Sensing for primary users before using the band is one possible approach to meet the non-interference constraint [9]. In order to provide a safety margin for the primary users against deep fades, the cognitive radios must sense the primary users' signals at very low SNRs [10]. Low $S N R$ signal detection problems arise in other contexts too. For instance, indoor GPS receivers need to operate well below the noise floor [11]. Also, researchers in the Search for Extra Terrestrial Intelligence (SETI) community are interested in the problem of detecting signals transmitted by space aliens extremely far away [12].

Fishman [13] showed that the radiometer is non-robust to uncertainties in the noise power below a certain $S N R$. This is true because the radiometer uses the average power as a test-statistic to perform detection and it ignores any structure associated with the signal. It was later shown that for signals without any structure all possible detection algorithms have $S N R$ thresholds (called $S N R$ walls), below which they fail to be robust to modeling uncertainties [14]. Structured signals like signals with pilot tones or cyclostationary signals contain features that can be exploited to distinguish them from noise [15], [16]. These robustness gains lower the $S N R$ wall, but cannot completely eliminate it. Uncertainty in the fading process combined with the uncertainty in the noise is the main reason for the non-robustness of signals with such features. At low SNRs the fading process blurs these sort of signal features, making them indistinguishable from noise.

In this paper, we construct signals with macroscale features, and show that these features can be robustly detected even in the presence of arbitrarily varying noise and fading processes with bounded delay spreads. The key idea is that if the scale of the feature is larger than the delay spread of the channel, then any fading process cannot completely blur the signal feature. So, with sufficient averaging the signal feature can be distinguished from noise. The main steps in the construction of these signals are as follows: we first divide time into blocks such that the length of each block is larger than the maximum delay spread of the channel. Then, we partition these blocks into two groups. Finally, two signals with different distributions are chosen and transmitted in each group respectively. An example of such a construction is a block-interleaved white Gaussian processes with distinct power levels.

The secondary users can partition the received samples into two groups corresponding to the two signal features. If the received signal is just noise, these two partitions look identical; otherwise these partitions will look distinct by construction. The idea is that the fading process cannot mix the signals within the two groups to an extent that they look identical. Therefore, we can robustly distinguish the noisy signal from noise alone.

Our previous results on $S N R$ walls suggested a tradeoff between the data rate achievable for the primary users and the robustness of secondary detection. We called this the capacityrobustness tradeoff [14]. The results in this paper show that it is possible to construct signals with infinite robustness (no $S N R$ wall) to the secondary user, while the primary user 
is still operating arbitrarily close to its capacity. This makes the capacity-robustness tradeoff uninteresting. In this paper we show that there seems instead to be a tradeoff between the primary data rate and the delay (sensing time) for the secondary user. We call this the capacity-delay tradeoff.

The idea of macroscale features has been applied in other scenarios [17]. Here a primary user wants to robustly identify potential secondary users interfering with its transmissions. In this case each secondary user is given an unique macroscale code sequence that can be robustly detected by the primary user.

The rest of the paper is organized as follows. We formulate the detection problem as a robust hypothesis test and review our previous $S N R$ wall results in Section II. We describe the idea of macroscale features by giving examples of signals with macroscale features in Section III. An explicit construction of a Gaussian mixture signal, a common-randomness based algorithm to robustly detect this signal in noise and its robustness analysis is given in Section III. The capacity-delay tradeoff for the Gaussian example is derived in Section IV. Finally, we end the paper by giving some concluding remarks in Section V.

\section{PROBLEM FORMULATION AND BACKGROUND REVIEW}

For mathematical convenience we work in discrete time. The problem of detecting the presence/absence of signals in noise can be modeled as a binary hypothesis test between the following hypotheses:

$$
\begin{aligned}
& \mathcal{H}_{0}: Y[n]=W[n] \\
& \mathcal{H}_{1}: Y[n]=H(X)[n]+W[n],
\end{aligned}
$$

for $n=1,2, \cdots, N$, where $N$ is the number of samples collected for detection. Here, $Y[n], X[n], W[n]$ and $H(\cdot)$ denote the received signal, the transmitted signal, noise at time $n$, and the linear time-varying fading operator respectively. We are interested in designing detection algorithms that perform well in the presence of modeling uncertainties in the noise and fading processes. The results in this paper show the existence of signals that can be robustly detected in the presence of modeling uncertainties. Since we are aiming to prove positive robustness results, we make minimal structural assumptions about the uncertainty models. This is opposite to the approach in our previous papers [14], [18], in which we assumed structured uncertainty models. The approach in our previous papers is justified because we showed that robustness for most signals is impossible even under structured uncertainty models, and the robustness performance would be even worse under unstructured ones.

In this paper we model the noise as an arbitrarily varying sequence having an empirical second moment. That is, $\frac{1}{N} \sum_{n=1}^{N}|W[n]|^{2}=: \sigma^{2}$, for some unknown $\sigma>0$. Further, we assume that there exist known constants $N_{0}>0$, and $C_{1}>0$, such that

$$
\frac{1}{N} \sum_{n=1}^{N}|W[n]|^{4}<C_{1}, \quad \forall N>N_{0} .
$$

For notational convenience, denote the set of all noise vectors satisfying these conditions by $\mathbb{W}$.

We model the fading process as a finite-tap linear timevarying filter, i.e., $H(X)[n]=\sum_{l=0}^{L-1} h_{l}[n] X[n-l]$, for some finite constant $L>0$. Further, assume that the sequences $\left\{h_{l}[n]\right\}_{n=1}^{\infty}, l=0,1, \cdots, L-1$ are arbitrarily varying but satisfy $\frac{1}{N} \sum_{n=1}^{N} \sum_{l=0}^{L-1}\left|h_{l}[n]\right|^{2}=1$. This assumption is made for mathematical convenience. Typically, fading leads to low SNRs, but in this paper we assume that the signal power is low and the fading process is normalized to have unit power on average. We also assume that there exist known constants $N_{1}>0$ and $C_{2}>0$ such that

$$
\frac{1}{N} \sum_{n=1}^{N} \sum_{l=0}^{L-1}\left|h_{l}[n]\right|^{4} \leq C_{2}, \quad \forall N>N_{1} .
$$

Denote the set of fading operators satisfying these constraints by $\mathbb{H}$. Any detection algorithm can be viewed as a function $F: \mathbb{R}^{N} \rightarrow\{0,1\}$ that maps the $N$-dimensional received signal vector $\mathbf{Y}:=(Y[1], Y[2], \cdots, Y[N])$ onto the set $\{0,1\}$. Here ' 0 ' (' 1 ') stands for the detectors decision that the signal is absent (present). For a fixed noise vector $\mathbf{W} \in \mathbb{W}$, and fading operator $H(\cdot) \in \mathbb{H}$, the error probabilities are defined as

$$
\begin{aligned}
P_{F A}(\mathbf{W}) & =\mathbb{E}\left[\mathbf{1}_{\{F=1\}} \mid \mathcal{H}_{0}\right] \\
P_{M D}(\mathbf{W}, H) & =\mathbb{E}\left[\mathbf{1}_{\{F=0\}} \mid \mathcal{H}_{1}\right],
\end{aligned}
$$

where the expectation is taken over the randomness in the transmitted signal $\mathbf{X}$.

Definition 1: A decision strategy robustly achieves a given target probability of false alarm, $P_{F A}$, and probability of missed detection, $P_{M D}$ if the algorithm satisfies

$$
\begin{aligned}
\sup _{\mathbf{W} \in \mathbb{W}} P_{F A}(\mathbf{W}) & \leq P_{F A} \\
\sup _{\mathbf{W} \in \mathbb{W}, H \in \mathbb{H}} P_{M D}(\mathbf{W}, H) & \leq P_{M D} .
\end{aligned}
$$

The aim of this paper is to construct signals, $\{X[n]\}_{n=1}^{N}$, for which there exist detection algorithms that can robustly achieve any pair of error probabilities $\left(P_{F A}, P_{M D}\right)(0<$ $P_{F A} \leq 1$, and $\left.0<P_{M D} \leq 1\right)$ as long as the sensing time $N$ is sufficiently large.

We now review some of our previous results on robust detection of low $S N R$ signals. The reader is encouraged to read [14], [18], [19] for complete details. The main result of these papers was that in the presence of modeling uncertainties, most commonly occurring signals cannot be robustly distinguished from noise below certain $S N R$ thresholds. We called these thresholds the "SNR walls" for the detectors. We showed that if the $S N R$ is below the $S N R$ wall for the detector then any pair of error probabilities $0<P_{F A}<0.5$, $0<P_{M D}<0.5$ cannot be robustly achieved, no matter how long we sense for the signal.

The key reason for the SNR-wall phenomenon is that the set of distributions under both hypotheses completely overlap at low SNRs. This is pictorially shown in Figure 1(a) for the case of signals without any features. The shaded regions in the figure show the set of cumulative distribution functions ( $\mathrm{CDFs})$ 
under both hypotheses. The picture on the left in Figure 1(a) corresponds to the case when the operating SNR is higher than the SNR wall. In this case, there is no overlap between the set of CDFs under both hypotheses. So, we can use any quantile detector $^{1}$ to differentiate between the two hypotheses. Now, if we lower the SNR below the SNR wall, there is complete overlap between the two sets of CDFs (see the picture on the right in Figure 1(a)). Hence robust detection is impossible, irrespective of the number of samples collected.

For signals with features like sinusoidal pilot tones, and cyclostationary features ${ }^{2}$ robust detection is relatively easier. However, even for these signals robust detection becomes impossible below a certain SNR (See Figure 1(b)). We consider a simple example to illustrate the key reason behind the nonrobustness of such signals. Define,

$$
X_{1}[n]=\left\{\begin{array}{ccc} 
\pm \sqrt{2 P} & \text { w.p. } \frac{1}{2} & \text { if } n \text { is odd, } \\
0 & & \text { if } n \text { is even. }
\end{array},\right.
$$

$X_{1}[n]$ has zeros in all the even time samples and iid Bernoulli $\pm \sqrt{2 P}$ random variables in the odd time samples. This is an example of a cyclostationary signal. Detectors for cyclostationary signals were first proposed by William Gardner [20], [21]. It was shown in [18] that such feature detectors are nonrobust to uncertainties in noise and fading processes and they have an $S N R$ wall below which robust detection is impossible. The signal $X_{1}[n]$ in (4) was used as an example to propose a general signal processing technique called run-time noise calibration [19]. This technique improves detector robustness by lowering the $S N R$ wall. However, it does not eliminate the $S N R$ wall completely.

The reason for the non-robustness of detecting $X_{1}[n]$ is that simple two-tap fading filters are sufficient to destroy the signal feature. For instance, even a pure delay block-fading process introduces an $S N R$ wall [19]. Furthermore, if we allow for arbitrarily varying $L \geq 2$ tap fading processes, it is clear that the 2-tap filter $h_{0}[n]=\frac{1}{2}, h_{1}[n]=\frac{1}{2}$ largely destroys the feature in the signal $X_{1}[n]$.

\section{Signals with Macroscale features}

The cyclostationary signal example in the previous section suffered from SNR wall limitations because the scale of the signal feature was comparable to the scale of the fading process. Our goal in this section is to construct classes of signals that can be robustly detected in presence of uncertain noise and fading processes. The key idea is to introduce macroscale features in signals, which in turn enable robust detection even in the presence of uncertainties.

Let $X_{2}[n]$ be a discrete time signal given by

$$
X_{2}[n]=\left\{\begin{array}{cccc} 
\pm \sqrt{2 P} & \text { w.p. } \frac{1}{2} & \text { if }\left\lceil\frac{n}{K}\right\rceil & \text { is odd } \\
0 & & \text { if }\left\lceil\frac{n}{K}\right\rceil & \text { is even. }
\end{array}\right.
$$

\footnotetext{
${ }^{1}$ This detector computes the empirical estimate of a given quantile and compares it to a threshold.

${ }^{2}$ Cyclostationary signals are random processes with periodic second-order statistics, i.e., the mean and autocorrelation functions are periodic in time.
}
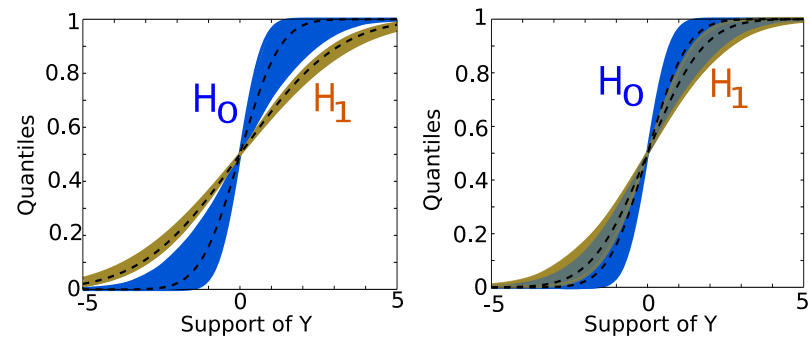

(a)
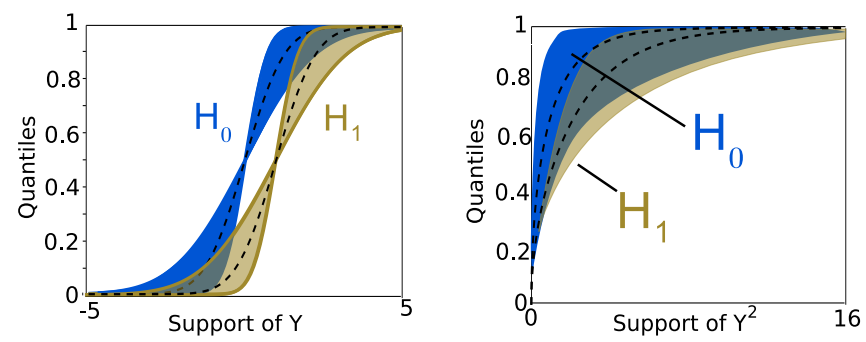

(b)

Fig. 1. The set of CDFs under both hypotheses are plotted for two example signals. In (a), the primary signal is chosen to be a zero-mean Gaussian. The picture on the left in (a) corresponds to a case where the operating SNR is larger than the SNR wall and hence the set of CDFs do not overlap. The picture on the right in (a) corresponds the case when the operating SNR is lower than the SNR wall. In this case the set of CDFs completely overlap. In (b), the primary signal is a known constant (sinusoidal pilot tone at zero frequency). The picture on the left in (b) shows the CDFs of both hypotheses. There is partial overlap, but the median is always separated and hence robust detection is possible at arbitrarily low SNRs. The picture on the right in (b) shows the $\mathrm{CDF}$ of the square of the samples $\left(Y^{2}[n]\right)$ under both hypotheses. It is clear that there is complete overlap in the set of CDFs under both hypotheses. This shows that squaring the signal destroys the signal feature.

for some $K \gg L$. The signal, $X_{2}[n]$ can be thought of as a vector version of $X_{1}[n]$. Time is divided into blocks of size $K$, the odd numbered blocks contain iid Bernoulli $\pm \sqrt{2 P}$ random variables, and the even numbered blocks have zeros. We call the former 'data' blocks and the latter 'silence' blocks.

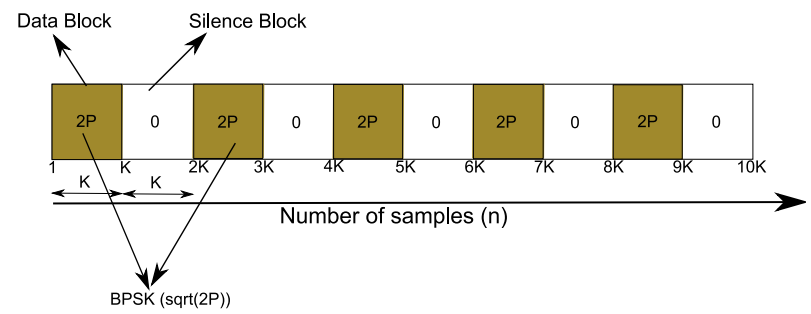

Fig. 2. This figure pictorially shows the signal described in Equation (5). The solid regions correspond to the "data" blocks, which contain iid $\operatorname{BPSK}( \pm \sqrt{2 P})$ signal samples, and the empty regions correspond to the "silence" blocks, and they contain zeros.

$X_{1}[n]$ and $X_{2}[n]$ are both cyclostationary signals, and they exhibit cyclostaionary features. The key difference is that the scale of the feature in $X_{2}[n]$ is much larger than the scale of the feature in $X_{1}[n]$. This is true because the width of the ON/OFF periods in $X_{2}[n]$ is $K$, which is larger than the 
width of the ON/OFF periods in $X_{1}[n]$. For this reason, we say that $X_{2}[n]$ has a macroscale feature (relative to the feature of $\left.X_{1}[n]\right)$.

If $K>L$, the scale of the feature in $X_{2}[n]$ is larger than the span of taps in the fading process, and it is clear that even an arbitrarily varying fading process cannot completely mix the 'data' blocks and the 'silence' blocks. So, even after fading, the signal will have some parts that are guaranteed to be silent (zero signal power) and some parts containing data (non-zero signal power). Moreover, the silent blocks occur periodically in time. This means that we can run a detector that searches for these periodic silences and uses these silence periods to learn the noise statistics. Note that the number of alternatives in the search does not grow exponentially with the sensing time, and hence our algorithm will successfully detect the signal given a sufficiently long sensing duration.

The robustness result for the example discussed above is not limited to the case where the delay spread $L$ is an absolute cutoff on the channel response. These results continue to hold for fading processes with infinite impulse response (IIR), as long as the fading coefficients satisfy the following property: at any given instance of time $n$, the first $L$ fading coefficients $h_{l}[n], l=0,1, \cdots, L-1$ contain more than half the total energy. In other words we are assuming that most of the energy in the received signal is coming from paths than have a delay falling within the first $L$ filter taps. This modeling assumption on the fading process is not unrealistic if we choose $L$ sufficiently large. It is easy to see that under this assumption the example $X_{2}[n]$ can still be robustly distinguished from noise. To keep the analysis simple, we assume that $L$ is an absolute cutoff in the rest of the paper.

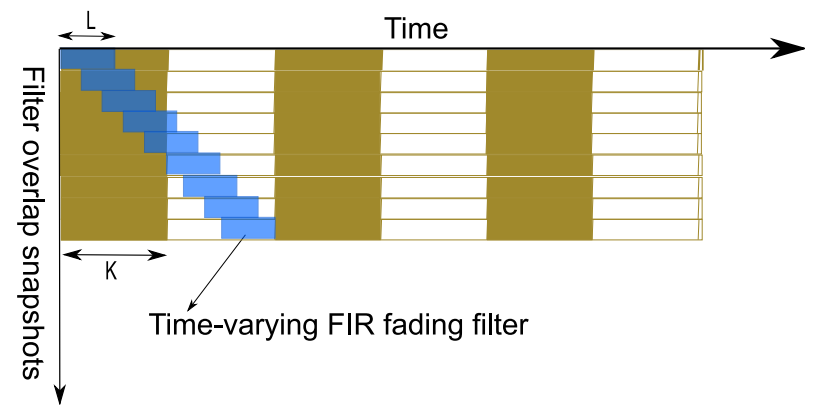

Fig. 3. This figure shown the output of the signal $X_{2}[n]$, when is it passed through a linear time-varying fading process with $L$ taps. From the figure it is clear that at certain time instances, the fading mixes with all zeros in the signal and so the output is also zero. Hence, in these time slots we can have access to noise even under hypothesis $\mathcal{H}_{1}$.

\section{A. Macroscale feature example: mixture of Gaussian signals}

The signal $X_{2}[n]$ given in (5) can robustly be detected in noise at arbitrarily low SNRs. However, this kind of signaling is wasteful because half the degrees of freedom are unused. We now give another example of a signal that contains macroscale features, and also makes better use of the degrees of freedom.
Define $\widetilde{X}_{2}[n]$ to be

$$
\widetilde{X}_{2}[n]=\left\{\begin{array}{ll}
\mathcal{N}(0, P+\epsilon) & \text { if }\left\lceil\frac{n}{K}\right\rceil \text { is odd, } \\
\mathcal{N}(0, P-\epsilon) & \text { if }\left\lceil\frac{n}{K}\right\rceil \text { is even. }
\end{array},\right.
$$

for some $0<\epsilon<P$. This signals is obtained by interleaving two iid Gaussian signals with power $P+\epsilon$ and $P-\epsilon$ respectively. The detection algorithm in this case is to compute the difference in the average power between the odd numbered blocks and the even numbered blocks. If the received signal is stationary noise, then this difference converges to zero as $N$ increases to infinity. If the received signal contains $\widetilde{X}_{2}[n]$, then the difference in powers converges to a non-zero constant proportional to $\epsilon$. Hence, this signal can be robustly distinguished from stationary noise.

However, if the noise itself has a time-varying variance then it is possible that the difference in the average power between the odd numbered blocks and the even numbered blocks is non-zero even under hypothesis $\mathcal{H}_{0}$. So, robust detection is not guaranteed at low SNRs. The possibility of noise having timevarying variance is not unrealistic because noise also contains interference, which is due to man-made signals transmitted by other users over the wireless medium.

One way to overcome this problem is to choose a complex signature that does not occur either in nature or in other licensed systems. This is analogous to the idea proposed by Carl Sagan that space-aliens would signal to us by modulating the signal with prime numbers that could not occur by any natural process unlike pure pilot tones [22]. The complex signature is used to choose the blocks to transmit a Gaussian signal with power $P+\epsilon$, while the remaining blocks are used to transmit a Gaussian signal with power $P-\epsilon$. To model this different level of complexity, we make a technical assumption of common randomness to capture codes that are effectively random when viewed from the perspective of natural effects. Specifically, we assume common randomness between the secondary detector and the primary transmitter. This allows the secondary detector to know the random partitioning of blocks created by the primary transmitter. The detailed description of the construction of a common-randomness based signal with macroscale features is given below.

Let $X[n]$ denote the signal we are trying to construct. Divide time into blocks of length $K=M+L-1$ for some $M>0$. Rewrite the sequence $\{X[n]\}_{n=1}^{\infty}$ as $\left\{\mathbf{X}_{i}\right\}_{i=1}^{\infty}$, where $\mathbf{X}_{i} \in \mathbb{R}^{1 \times K}$ is a $K$ dimensional row vector given by $\mathbf{X}_{i}=(X[(i-1) K+1], X[(i-1) K+2], \cdots, X[(i-1) K+$ $K])$. Let $\left\{B_{i}\right\}_{i=1}^{\infty}$ be a sequence of iid Bernoulli- $\frac{1}{2}$ random variables taking values in the set $\{0,1\}$. Think of this Bernoulli sequence as labeling the sequence $\left\{\mathbf{X}_{i}\right\}_{i=1}^{\infty}$ by '1's and ' 0 's.

Given the Bernoulli sequence $\left\{B_{i}\right\}_{i=1}^{\infty}$, define

$$
\mathbf{X}_{i}=\left\{\begin{array}{lll}
\mathcal{N}\left(\mathbf{0},(P+\epsilon) \mathbf{I}_{K \times K}\right) & \text { if } & B_{i}=1 \\
\mathcal{N}\left(\mathbf{0},(P-\epsilon) \mathbf{I}_{K \times K}\right) & \text { if } & B_{i}=0
\end{array},\right.
$$

where $\mathcal{N}\left(\mathbf{m}, \Lambda_{K \times K}\right)$ denotes a $K$-dimensional Gaussian random vector with mean $\mathbf{m}$ and covariance matrix $\Lambda_{K \times K}$. Here $\epsilon$ is chosen such that $0<\epsilon<P$, where $P$ is the average power of the signal. Intuitively, the signal $\left\{\mathbf{X}_{i}\right\}_{i=1}^{\infty}$ is a mixture of 
two iid random Gaussian processes, one with power $(P+\epsilon)$ and another with power $(P-\epsilon)$. The choice of the block length $K$ ensures that fading cannot completely mix the two Gaussian processes.

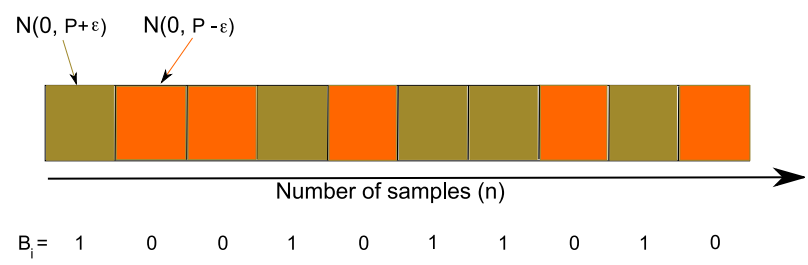

Fig. 4. This figure pictorially shows the signal described in Equation (7) The realization of the bit sequence $B_{i}$ decides the signal transmitted in block $i$. If $B_{i}=1$, then a $\mathcal{N}(0, P+\epsilon)$ signal is transmitted in block $i$, and if $B_{i}=0$, then a $\mathcal{N}(0, P-\epsilon)$ signal is transmitted in block $i$.

Assume that the same Bernoulli sequence $\left\{B_{i}\right\}_{i=1}^{\infty}$ is also available at the detector. This can be thought of as common randomness between the primary transmitter and the secondary detector. Given this common randomness, the detection algorithm can be described as follows:

- For any $K$-dimensional vector $\mathbf{X}:=$ $(X[1], X[2], \cdots, X[K]) \in \mathbb{R}^{1 \times K}, K>L$, define the function $\kappa(\mathbf{X}):=\frac{1}{K-L+1} \sum_{k=L}^{K}|X[k]|^{2}$. This function computes the empirical power (excluding the first $L-1$ samples) of the vector $\mathbf{X}$.

- Assume that $N$ is an integer multiple of $K$, i.e., $N=$ $K \cdot G$ for some integer $G>0$. Rewrite the received sequences $\{Y[n]\}_{n=1}^{N}$ as a sequence of $K$-dimensional vectors $\left\{\mathbf{Y}_{i}\right\}_{i=1}^{G}$, where $\mathbf{Y}_{i}=(Y[(i-1) K+1], Y[(i-$ 1) $K+2], \cdots, Y[(i-1) K+K])$.

- Compute

$$
\begin{aligned}
T_{1} & :=\frac{1}{G} \sum_{i=1}^{G} B_{i} \cdot \kappa\left(\mathbf{Y}_{i}\right) \\
T_{2} & :=\frac{1}{G} \sum_{i=1}^{G}\left(1-B_{i}\right) \cdot \kappa\left(\mathbf{Y}_{i}\right)
\end{aligned}
$$

$T_{1}$ and $T_{2}$ are the empirical average of the received signal powers in the blocks labeled ' 1 ' and ' 0 ' respectively.

- The detector is given by

$$
T\left(\left\{\mathbf{Y}_{i}\right\}_{i=1}^{G}\right):=T_{1}-T_{2} \underset{\mathcal{H}_{0}}{\stackrel{\mathcal{H}_{1}}{\gtrless}} \lambda,
$$

where $\lambda>0$ is the detection threshold.

The intuitive justification for choosing the test-statistic in (9) is as follows: if the signal is present, the difference in powers in the blocks labeled ' 1 ' and the blocks labeled ' 0 ' converges in probability to a non-zero number as $G \rightarrow \infty$. On the other hand, if the signal is absent, this difference converges to zero as $G \rightarrow \infty$.

\section{B. Robustness analysis}

Theorem 1: Consider the robust hypothesis testing problem in (1). Assume that the sensing time can be written as $N=$

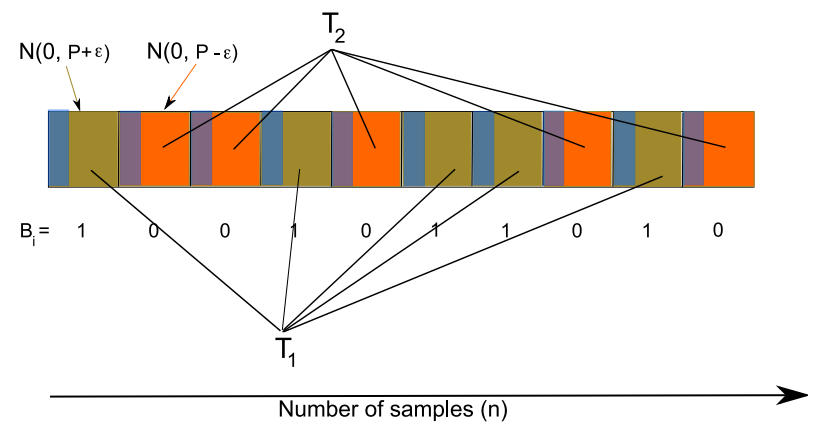

Fig. 5. This figure pictorially shows the detection algorithm described in Equation (9).

$K \cdot G$, for some positive integers $K$ and $G$. Let $H(X)[n]=$ $\sum_{l=1}^{L-1} h_{l}[n] X[n-l]$, for some integer $L>0$. Assume that the fading and noise processes satisfy the following constraints.

- $\frac{1}{G \cdot K} \sum_{n=1}^{G \cdot K}|W[n]|^{2}=\sigma^{2}$ for some unknown $\sigma>0$.

- $\frac{1}{G \cdot K} \sum_{n=1}^{G \cdot K} \sum_{l=0}^{L-1}\left|h_{l}[n]\right|^{2}=1$.

- There exist known constants $G_{0}>0$, and $C_{i}>0, i=$ $1,2,3$, such that for all $G>G_{0}$, we have

$$
\begin{array}{r}
\frac{1}{G \cdot K} \sum_{n=1}^{G \cdot K}|W[n]|^{4}<C_{1} \\
\frac{1}{G \cdot K} \sum_{n=1}^{G \cdot K} \sum_{l=0}^{L-1}\left|h_{l}[n]\right|^{4}<C_{2} \\
\frac{1}{G \cdot K} \sum_{n=1}^{G \cdot K}\left(\sum_{l=0}^{L-1}\left|h_{l}[n]\right|^{2}\right)|W[n]|^{2}<C_{3} .
\end{array}
$$

Let $\left\{B_{i}\right\}_{i=1}^{G}$ be a sequence of Bernoulli- $\frac{1}{2}$ iid random variables taking values in the set $\{0,1\}$. Rewrite the signal samples $\{X[n]\}_{n=1}^{N}$ as $\left\{\mathbf{X}_{i}\right\}_{i=1}^{G}$, where $\mathbf{X}_{i}:=(X[(i-1) K+$ $1], \cdots, X[(i-1) K+K])$, and

$$
\mathbf{X}_{i}=\left\{\begin{array}{lll}
\mathcal{N}\left(\mathbf{0},(P+\epsilon) \mathbf{I}_{K \times K}\right) & \text { if } & B_{i}=1 \\
\mathcal{N}\left(\mathbf{0},(P-\epsilon) \mathbf{I}_{K \times K}\right) & \text { if } & B_{i}=0
\end{array},\right.
$$

where $0<\epsilon<P$.

Let $K>L$. Consider the detection algorithm in (9). Define,

$$
\begin{aligned}
P_{F A}(G) & :=\mathcal{P}\left(T\left(\left\{\mathbf{Y}_{i}\right\}_{i=1}^{G}\right)>\lambda \mid \mathcal{H}_{0}\right) \\
P_{M D}(G) & :=\mathcal{P}\left(T\left(\left\{\mathbf{Y}_{i}\right\}_{i=1}^{G}\right)<\lambda \mid \mathcal{H}_{1}\right) .
\end{aligned}
$$

Then, for any given choice of $1 \geq P_{F A}>0,1 \geq P_{M D}>0$, and $P>0$, there exists a $G$ such that $P_{F A}(G) \leq P_{F A}$, $P_{M D}(G) \leq P_{M D}$. Hence, the detection algorithm in (9) is robust to uncertainties for all $P>0$.

Proof: From Equations (9) and (12),

$$
P_{F A}(G)=\mathcal{P}\left(T_{1}-T_{2}>\lambda \mid \mathcal{H}_{0}\right)
$$


By the definition of $T_{1}$ in (8),

$$
\begin{aligned}
\mathbb{E}\left[T_{1} \mid \mathcal{H}_{0}\right] & =\frac{1}{G} \sum_{i=1}^{G} \mathbb{E}\left[B_{i} \cdot \kappa\left(\mathbf{Y}_{i}\right) \mid \mathcal{H}_{0}\right] \\
& =\frac{1}{G} \sum_{i=1}^{G} \mathbb{E}\left[B_{i} \cdot \kappa\left(\mathbf{W}_{i}\right)\right] \\
& =\frac{1}{2}\left[\frac{1}{G} \sum_{i=1}^{G} \kappa\left(\mathbf{W}_{i}\right)\right],
\end{aligned}
$$

where $\mathbf{W}_{i}=(W[(i-1) K+1], \cdots, W[(i-1) K+K])$. Similarly, we can show that

$$
\mathbb{E}\left[T_{2} \mid \mathcal{H}_{0}\right]=\frac{1}{2}\left[\frac{1}{G} \sum_{i=1}^{G} \kappa\left(\mathbf{W}_{i}\right)\right] .
$$

From (14) and (15), we have $\mathbb{E}\left[T_{1}-T_{2} \mid \mathcal{H}_{0}\right]=0$. So, from Chebyshev's inequality [23] we get

$$
\mathcal{P}\left(\left|T_{1}-T_{2}\right|>\lambda \mid \mathcal{H}_{0}\right) \leq \frac{\operatorname{Var}\left[T_{1}-T_{2} \mid \mathcal{H}_{0}\right]}{\lambda^{2}}
$$

Using (16) in (13),

$$
\begin{aligned}
P_{F A}(G) & \leq \mathcal{P}\left(\left|T_{1}-T_{2}\right|>\lambda \mid \mathcal{H}_{0}\right) \\
& \leq \frac{\operatorname{Var}\left[T_{1}-T_{2} \mid \mathcal{H}_{0}\right]}{\lambda^{2}}
\end{aligned}
$$

We now compute the expressions under hypothesis $\mathcal{H}_{1}$.

$$
\begin{aligned}
\mathbb{E}\left[T_{1} \mid \mathcal{H}_{1}\right] & =\mathbb{E}\left[\frac{1}{G} \sum_{i=1}^{G} B_{i} \cdot \kappa\left(\mathbf{Y}_{i}\right) \mid \mathcal{H}_{1}\right] \\
& =\left[\frac{1}{G} \sum_{i=1}^{G} \mathbb{E}\left[B_{i} \cdot \kappa\left(\mathbf{Y}_{i}\right) \mid \mathcal{H}_{1}\right]\right] .
\end{aligned}
$$

The expectation on the RHS of (18) can be simplified as

$$
\begin{aligned}
& \mathbb{E}\left[B_{i} \cdot \kappa\left(\mathbf{Y}_{i}\right) \mid \mathcal{H}_{1}\right] \\
= & \mathbb{E}\left[B_{i} \cdot \frac{1}{K-L+1} \sum_{k=L}^{K}|Y[(i-1) K+k]|^{2} \mid \mathcal{H}_{1}\right] \\
= & \frac{1}{K-L+1} \sum_{k=L}^{K} \mathbb{E}\left[B_{i}|Y[(i-1) K+k]|^{2} \mid \mathcal{H}_{1}\right] .
\end{aligned}
$$

Again, the expectation within the summation in the above equation can be simplified using $\tilde{k}=(i-1) K+k$ as

$$
\begin{aligned}
& \mathbb{E}\left[B_{i}|Y[\tilde{k}]|^{2} \mid \mathcal{H}_{1}\right] \\
= & \mathbb{E}\left[B_{i}\left|\sum_{l=0}^{L-1} h_{l}[\tilde{k}] X[\tilde{k}-l]+W[\tilde{k}]\right|^{2}\right] \\
= & \frac{1}{2} \mathbb{E}\left[\left|\sum_{l=0}^{L-1} h_{l}[\tilde{k}] X[\tilde{k}-l]+W[\tilde{k}]\right|^{2} \mid X \sim \mathcal{N}(0, P+\epsilon)\right] \\
= & \frac{1}{2}\left[\left((P+\epsilon) \sum_{l=0}^{L-1}\left|h_{l}[\tilde{k}]\right|^{2}\right)+|W[\tilde{k}]|^{2}\right],
\end{aligned}
$$

we get

$$
\begin{aligned}
& \mathbb{E}\left[T_{1} \mid \mathcal{H}_{1}\right] \\
= & {\left[\frac { 1 } { G } \sum _ { i = 1 } ^ { G } \left(\frac { 1 } { K - L + 1 } \sum _ { k = L } ^ { K } \frac { 1 } { 2 } \left\{\sum_{l=0}^{L-1}(P+\epsilon)\left|h_{l}[\tilde{k}]\right|^{2}\right.\right.\right.} \\
& \left.\left.\left.+|W[\tilde{k}]|^{2}\right\}\right)\right] .
\end{aligned}
$$

Similarly, we can also show that

$$
\begin{aligned}
& \mathbb{E}\left[T_{2} \mid \mathcal{H}_{1}\right] \\
= & {\left[\frac { 1 } { G } \sum _ { i = 1 } ^ { G } \left(\frac { 1 } { K - L + 1 } \sum _ { k = L } ^ { K } \frac { 1 } { 2 } \left\{\sum_{l=0}^{L-1}(P-\epsilon)\left|h_{l}[\tilde{k}]\right|^{2}\right.\right.\right.} \\
& \left.\left.\left.+|W[\tilde{k}]|^{2}\right\}\right)\right] .
\end{aligned}
$$

From (21) and (22), we get

$$
\begin{aligned}
& \mathbb{E}\left[T_{1}-T_{2} \mid \mathcal{H}_{1}\right] \\
= & \epsilon \cdot\left[\frac{1}{G \cdot(K-L+1)} \sum_{i=1}^{G} \sum_{k=L}^{K} \sum_{l=0}^{L-1}\left|h_{l}[(i-1) K+k]\right|^{2}\right] \\
=: & \epsilon \cdot \Theta .
\end{aligned}
$$

From the definition of $\Theta$ above, it is clear that $\Theta$ is the empirical average power of the fading coefficients. From the assumption in the statement of the theorem, it is clear that $\Theta \rightarrow 1$ as $G \rightarrow \infty$. Again, using Chebyshev's inequality, we get

$$
\mathcal{P}\left(\left|\left(T_{1}-T_{2}\right)-\epsilon \cdot \Theta\right|>\tilde{\lambda} \mid \mathcal{H}_{1}\right) \leq \frac{\operatorname{Var}\left[T_{1}-T_{2} \mid \mathcal{H}_{1}\right]}{\tilde{\lambda}^{2}},
$$

where $\tilde{\lambda}=\epsilon \cdot \Theta-\lambda$. Now, the probability of mis-detection is given by

$$
\begin{aligned}
P_{M D}(G) & =\mathcal{P}\left(T_{1}-T_{2}<\lambda \mid \mathcal{H}_{1}\right) \\
& =\mathcal{P}\left(\left(T_{1}-T_{2}\right)-\epsilon \cdot \Theta<\lambda-\epsilon \cdot \Theta \mid \mathcal{H}_{1}\right) \\
& =\mathcal{P}\left(\left(T_{1}-T_{2}\right)-\epsilon \cdot \Theta<-\tilde{\lambda} \mid \mathcal{H}_{1}\right) \\
& \leq \mathcal{P}\left(\left|\left(T_{1}-T_{2}\right)-\epsilon \cdot \Theta\right|>\tilde{\lambda} \mid \mathcal{H}_{1}\right) \\
& \leq \frac{\operatorname{Var}\left[T_{1}-T_{2} \mid \mathcal{H}_{1}\right]}{\tilde{\lambda}^{2}}
\end{aligned}
$$

From (16) and (24), it follow that $P_{F A}(G)$ and $P_{M D}(G)$ can be made arbitrarily small if $\operatorname{Var}\left[T_{1}-T_{2} \mid \mathcal{H}_{0}\right]$ and $\operatorname{Var}\left[T_{1}-\right.$ $\left.T_{2} \mid \mathcal{H}_{1}\right]$ decrease to zero as $G$ increases to $\infty$. This is proved in the following Lemma.

Lemma 1: For all $G>G_{0}$,

$$
\begin{aligned}
\operatorname{Var}\left[T_{1}-T_{2} \mid \mathcal{H}_{0}\right] & \leq \frac{\gamma_{1}}{G} \\
\operatorname{Var}\left[T_{1}-T_{2} \mid \mathcal{H}_{1}\right] & \leq \frac{\gamma_{2}}{G},
\end{aligned}
$$

where $\gamma_{1}$ and $\gamma_{2}$ are constants dependent only on $C_{i}, i=$ $1,2,3$.

Proof: The proof of this lemma is straightforward and it omitted here. 
Using the bounds in Lemma 1, we have

$$
\begin{aligned}
P_{F A}(G) & \leq \frac{\gamma_{1}}{G \cdot \lambda^{2}}, \\
P_{M D}(G) & \leq \frac{\gamma_{2}}{G \cdot(\epsilon \cdot \Theta-\lambda)^{2}}, \quad \forall G>G_{0} .
\end{aligned}
$$

\section{CAPACITY-DELAY TRADEOFF}

In the previous section, we have shown that signals with macroscale features can overcome $S N R$ wall limitations. The question we ask is whether the primary user has to pay a penalty for the improved robustness of the secondary user? We first look at the example considered in Section III-A. In this example, the primary signal is obtained by interleaving two random Gaussian sequences with different power levels. Assume that the channel from the primary transmitter to the primary receiver is an AWGN channel with noise power $\sigma_{p}^{2}$. We know that the capacity of this channel is given by $C_{p}=\frac{1}{2} \log \left(1+\frac{P}{\sigma_{p}^{2}}\right)$. However, the rate achieved at the primary receiver is the average of the rates achieved by the two Gaussian streams, i.e.,

$R_{p}:=\frac{1}{2}\left[\frac{1}{2} \log \left(1+\frac{P+\epsilon}{\sigma_{p}^{2}}\right)+\frac{1}{2} \log \left(1+\frac{P-\epsilon}{\sigma_{p}^{2}}\right)\right] \leq C_{p}$

The concavity- $\cap$ of the capacity function $C_{p}$ shows that the primary takes a hit in the data rate to its receivers in order to provide robustness guarantee to the secondary users. This rate penalty depends on the parameter $\epsilon$, and as $\epsilon \downarrow 0$, the rate penalty converges to zero. This suggests that the cost to the primary (in terms of loss in data rate) for providing robustness to the secondary can be made arbitrarily close to zero.

On the other hand, the parameter $\epsilon$ affects the sensing time (number of samples) required by the secondary user to robustly attain a given target $P_{F A}$ and $P_{M D}$. Eliminating $\lambda$ in (27), and using the fact that $\Theta \approx 1$ for large $G$, we get

$$
N=G \cdot K=K\left[\sqrt{\frac{\gamma_{1}}{P_{F A}}}+\sqrt{\frac{\gamma_{2}}{P_{M D}}}\right] \epsilon^{-2}
$$

Equation (29) tells us that the sensing delay at the secondary user monotonically increases as $\epsilon \downarrow 0$. Therefore, in the Gaussian mixture example there is tension between the primary data rate and the sensing delay at the secondary detector.

Comparing Equations (28) and (29) we can see that there is a tradeoff between the data rate $\left(R_{p}\right)$ achieved by the primary user and the sensing delay $(N)$ for the secondary user. We call this the capacity-delay tradeoff. An important question arising from this discussion is - whether there is a fundamental capacity-delay tradeoff? That is, does the primary user always have to take a hit in its data rate to provide sensing delay guarantees to the secondary users. The intuitive reasoning for the existence of a fundamental capacity-delay tradeoff is given below.

We know that the capacity-achieving output distribution is unique for most channels [24], [25]. Appealing to the continuity of mutual information this means that in most generic cases the capacity achieving input distribution is also unique. The results in this paper show that the secondary user can robustly detect the primary user if the primary signal is a mixture of two distinct distributions. So, if the primary user is operating close to capacity using an input signal that is a mixture of two distributions, then both these distinct distributions must be close to the unique capacityachieving input distribution. Hence by continuity, the two output distributions at the secondary receiver must also be close to each other. Hence, to distinguish between these two output distributions, the sensing delay at the secondary must be large. So, as the primary rate approaches its channel capacity, the sensing delay at the secondary must approach infinity.

\section{CONCLUDing REMARKS}

In this paper we considered the problem of robustly detecting the presence/absence of low $S N R$ signals under modeling uncertainties. We constructed signals with macroscale features and showed that these signals can be robustly detected in the presence of arbitrarily varying noise and fading processes. Hence, we have shown the existence of signals that overcome $S N R$ walls. This result is important as most commonly used communication signals were known to suffer from $S N R$ wall limitations.

An explicit construction of a mixture of iid Gaussian streams was given in this paper. For this signal, a simple detection algorithm was proposed that was shown to robustly detect this signal under uncertain noise and fading processes. The following observations can be drawn from the Gaussian mixture example:

- Due to the interleaving of two streams with different powers, the primary data rate is less than the capacity, which can be achieved by Gaussian signaling at power $P$. This hit in the primary data rate increases as $\epsilon$ increases.

- Even though the average power of the signal is $P$, there are local power fluctuations in the signal. This is because the signal is obtained by interleaving two streams, one with power $(P+\epsilon)$ and the other with power $(P-\epsilon)$. Because of these fluctuations, the delay from the primary transmitter to the primary receiver also increases.

- Finally, the sensing time for the secondary user also depends on the difference in powers between the two streams $(\epsilon)$. Large values of $\epsilon$ lead to smaller values of the secondary sensing time.

These observations suggest that there is a tradeoff between the primary data rate, delay from the primary transmitter to the primary receiver and the sensing delay to the secondary detector. Whether the tradeoff between these quantities is fundamental, or it is an artifact of this particular example is unknown. However, we believe that there is a fundamental tradeoff between the primary data rate and the sensing delay to the secondary user.

\section{ACKNOWLEDGEMENTS}

We thank the National Science Foundation (grants ANI326503, CNS-403427, CCF-729122), and Sumitomo Electric for their support. 


\section{REFERENCES}

[1] R. Price and N. Abramson, "Detection theory," IEEE Trans. Inform. Theory, vol. 7, pp. 135-139, July 1961.

[2] D. Slepian, "Some comments on the detection of Gaussian signals in Gaussian noise," IEEE Trans. Inform. Theory, vol. 4, pp. 65-68, June 1958.

[3] D. Middleton, "On the detection of stochastic signals in additive normal noise - part I," IEEE Trans. Inform. Theory, vol. 3, pp. 86-121, June 1957.

[4] D. Eaddy, T. Kadota, and J. Seery, "On the approximation of the optimum detector by the energy detector in detection of colored gaussian signals in noise," IEEE Trans. Acoust., Speech, Signal Processing, vol. 32, pp. 661-664, June 1984.

[5] FCC, "Et docket no. 03-237," Nov. 2003. [Online]. Available: http://hraunfoss.fcc.gov/edocs_public/attachmatch/FCC-03-289A1.pdf

[6] R. W. Broderson, A. Wolisz, D. Cabric, S. M. Mishra, and D. Willkomm. (2004) White paper: Corvus: A cognitive radio approach for usage of virtual unlicensed spectrum. [Online]. Available: http://bwrc.eecs.berkeley.edu/Research/MCMA/CR_White_paper_final1.pdf

[7] FCC, “Et docket no. 03-322," Dec. 2003. [Online]. Available: http://hraunfoss.fcc.gov/edocs_public/attachmatch/FCC-03-322A1.pdf

[8] J. Mitola, "Software radios: Survey, critical evaluation and future directions," IEEE Aerosp. Electron. Syst. Mag, vol. 8, pp. 25-36, Apr. 1993.

[9] C. R. Stevenson, C. Cordeiro, E. Sofer, and G. Chouinard, "Functional requirements for IEEE 802.22 WRAN standard," Tech. Rep., September 2005. [Online]. Available: http://www.ieee802.org/22/

[10] A. Sahai, N. Hoven, and R. Tandra, "Some fundamental limits on cognitive radio," in Forty-second Allerton Conference on Communication, Control, and Computing, Monticello, IL, Oct. 2004.

[11] N. Agarwal et al., "Algorithms for GPS operation indoors and downtown," GPS Solutions, no. 6, pp. 149-160, 2002.

[12] C. Sagan, Communication with Extraterrestrial Intelligence. MIT Press, 1972.

[13] A. Sonnenschein and P. M. Fishman, "Radiometric detection of spreadspectrum signals in noise of uncertain power," IEEE Trans. Aerosp. Electron. Syst., vol. 28, pp. 654-660, July 1992.

[14] R. Tandra and A. Sahai, "SNR walls for signal detection," IEEE Journal on Selected Topics in Signal Processing, vol. 2, pp. 4 - 17, Feb. 2008.

[15] W. A. Gardner, Statistical Spectral Analysis: A Nonprobabilistic Theory. Englewood Cliffs, NJ: Prentice-Hall, 1987.

[16] _ Introduction to Random Processes with Applications to Signals and Systems. New York: Macmillan, 1985.

[17] G. Atia, A. Sahai, and V. Saligrama, "Spectrum enforcement and liability assignment in cognitive raidio systems," in Proc. of the 3rd IEEE International Symposium on New Frontiers in Dynamic Spectrum Access Networks, Oct. 2008.

[18] R. Tandra and A. Sahai, "SNR walls for feature detectors," in Proc. of 2nd IEEE International Symposium on New Frontiers in Dynamic Spectrum Access Networks, April 2007, pp. 559-570.

[19] - "Noise calibration, delay coherence and SNR walls for signal detection," in Proc. of the 3rd IEEE International Symposium on New Frontiers in Dynamic Spectrum Access Networks, Oct. 2008.

[20] W. Gardner, "Signal interception: A unifying theoretical framework for feature detection," IEEE Trans. Commun., vol. 36, pp. 897-906, Aug. 1988.

[21] _ "Signal interception: Performance advantages of cyclic-feature detectors," IEEE Trans. Commun., vol. 40, pp. 149-159, Jan. 1992.

[22] C. Sagan, Cosmos. Random House, 1980.

[23] R. Durrett, Probability: Theory and Examples, 3rd ed. Belmont: Duxbury Press, 2004.

[24] T. Cover and J. Thomas, Elements of Information Theory, 2nd ed. Wiley-Interscience, 2006.

[25] R. Gallager, Information Theory and Reliable Communication. Wiley, 1968. 